\title{
A Vector Flow Imaging Method for Portable Ultrasound Using Synthetic Aperture Sequential Beamforming
}

di lanni, Tommaso; Villagómez Hoyos, Carlos Armando; Ewertsen, Caroline; Kjeldsen, Thomas Kim; Mosegaard, Jesper; Nielsen, Michael Bachmann; Jensen, Jørgen Arendt

Published in:

I E E E Transactions on Ultrasonics, Ferroelectrics and Frequency Control

Link to article, DOI:

10.1109/TUFFC.2017.2742599

Publication date:

2017

Document Version

Peer reviewed version

Link back to DTU Orbit

Citation (APA):

di lanni, T., Villagómez Hoyos, C. A., Ewertsen, C., Kjeldsen, T. K., Mosegaard, J., Nielsen, M. B., \& Jensen, J. A. (2017). A Vector Flow Imaging Method for Portable Ultrasound Using Synthetic Aperture Sequential Beamforming. I E E E Transactions on Ultrasonics, Ferroelectrics and Frequency Control, 64(11), 1655-1665. https://doi.org/10.1109/TUFFC.2017.2742599

\section{General rights}

Copyright and moral rights for the publications made accessible in the public portal are retained by the authors and/or other copyright owners and it is a condition of accessing publications that users recognise and abide by the legal requirements associated with these rights.

- Users may download and print one copy of any publication from the public portal for the purpose of private study or research.

- You may not further distribute the material or use it for any profit-making activity or commercial gain

- You may freely distribute the URL identifying the publication in the public portal 


\title{
A Vector Flow Imaging Method for Portable Ultrasound using Synthetic Aperture Sequential Beamforming
}

\author{
Tommaso Di Ianni ${ }^{1}$, Carlos Armando Villagómez Hoyos ${ }^{1}$, Caroline Ewertsen ${ }^{2}$, Thomas Kim Kjeldsen ${ }^{3}$, Jesper \\ Mosegaard $^{3}$, Michael Bachmann Nielsen ${ }^{2}$, and Jørgen Arendt Jensen ${ }^{1}$ \\ ${ }^{1}$ Center for Fast Ultrasound Imaging, Department of Electrical Engineering, Technical University of Denmark, \\ Kongens Lyngby 2800, Denmark \\ ${ }^{2}$ Department of Radiology, Rigshospitalet, Copenhagen University Hospital, Copenhagen 2100, Denmark \\ ${ }^{3}$ Visual Computing Lab, Alexandra Institute, Aarhus 8200, Denmark
}

\begin{abstract}
This paper presents a vector flow imaging (VFI) method for the integration of quantitative blood flow imaging in portable ultrasound systems. The method combines directional transverse oscillation (TO) and synthetic aperture sequential beamforming (SASB) to yield continuous velocity estimation in the whole imaging region. Six focused emissions are used to create a high-resolution image (HRI), and a dual-stage beamforming approach is used to lower the data throughput between the probe and the processing unit. The transmit/receive focal points are laterally separated to obtain a TO in the HRI that allows for the velocity estimation along the lateral and axial directions using a phase-shift estimator. The performance of the method was investigated with constant flow measurements in a flow rig system using the SARUS scanner and a 4.1 MHz linear array. A sequence was designed with interleaved B-mode and flow emissions to obtain continuous data acquisition. A parametric study was carried out to evaluate the effect of critical parameters. The vessel was placed at depths from 20 to $40 \mathrm{~mm}$, with beam-to-flow angles of $65^{\circ}, 75^{\circ}$, and $90^{\circ}$. For the lateral velocities at $20 \mathrm{~mm}$, a bias between $-5 \%$ and $-6.2 \%$ was obtained, and the standard deviation (SD) was between $6 \%$ and $9.6 \%$. The axial bias was lower than $1 \%$ with a SD around $2 \%$. The mean estimated angles were $66.70 \pm 2.86^{\circ}, \mathbf{7 2 . 6 5} \pm 2.48^{\circ}$, and $89.13 \pm 0.79^{\circ}$ for the three cases. A proof-of-concept demonstration of the real-time processing and wireless transmission was tested in a commercial tablet obtaining a frame rate of 27 FPS and a data rate of $14 \mathrm{MB} / \mathrm{s}$. An in-vivo measurement of a common carotid artery of a healthy volunteer was finally performed to show the potential of the method in a realistic setting. The relative SD averaged over a cardiac cycle was $4.33 \%$.
\end{abstract}

Index Terms-Vector flow imaging, synthetic aperture, transverse oscillation, portable ultrasound.

\section{INTRODUCTION}

Noninvasive imaging of blood flow using ultrasound is extensively used in the clinic due to the high temporal resolution, the portability, and the low cost compared with other imaging modalities. Pocket-size devices have the potential to bring ultrasound out of the radiology department, increasing its use in other medical fields with improved diagnostic accuracy and cost-effectiveness [1]-[3]. As highlighted by Prinz and Voigt [1], however, limited flow imaging capabilities and the absence of the spectral Doppler prevent the possibility of quantitative flow measurements, and further improvements are needed.

Vector flow imaging (VFI) methods estimate the velocity vectors directly from the measured ultrasound data with no need for manual angle adjustments and allow for the quantitative analysis of the blood flow even in presence of fast and complex flow dynamics [4]-[6]. The estimated angle can be used to reduce the inter-observer variability in the detection of the peak systolic velocity (PSV) [7]-[9]. Integrating the VFI feature in a portable scanner would improve the operator's workflow and reduce the inter-observer variability due to the angle-independent velocity estimation [10].

A first VFI approach relies on the combination of velocity components measured along several directions to obtain the estimated vector [11]. The 2-D tracking of blood speckle patterns was proposed by Trahey et al. [12], and was combined with plane wave (PW) excitation [13]. PWs have also been used in a number of other VFI approaches [14], [15]. Jensen and Munk [16] and Anderson [17] proposed the introduction in the ultrasound field of a transverse oscillation (TO), i.e. an oscillation lateral to the direction of the beam, which creates a modulation in the received signals proportional to the lateral displacement. Therefore, the velocity can be estimated along the lateral and axial directions using a phase-shift estimator [18]. Liebgott et al. [19] and Sumi [20] synthesized a TO by using a synthetic aperture (SA) for the optimization of the lateral oscillation frequency. A directional TO (DTO) approach was also proposed by Jensen [21] using directional beamforming in the lateral direction.

Parallel techniques as PW and SA acquire an entire image after every emission and provide continuous data [13], [22]. As a result, the time of observation is solely limited by the non-stationarity of the flow, and long sequences can be used for the estimation to obtain low standard deviation (SD) and high frame rate. In addition, the clutter filter has reduced complexity. Nevertheless, these techniques require considerable computational resources and conventional implementations are not suitable for a portable scanner.

An alternative solution is provided by SA sequential beam- 
forming (SASB) [23]. In SASB, the data received by an aperture of $L$ transducers are first beamformed in a static point, and one single line is obtained per emission, referred to as lowresolution line (LRL). The first beamformer can be integrated into the transducer front-end to reduce the data throughput of the probe by a factor $L$ [24], [25]. The fixed focal position is considered as a virtual source (VS) [26]-[28], and a second beamformer is used to re-focus the LRLs and obtain an highresolution image (HRI) dynamically focused in transmit and receive. Due to the dual-stage beamforming, SASB alleviates the system requirements compared with conventional SA and enables the wireless transmission of the data with current WiFi technologies. The 1-D flow estimation using SASB has been previously demonstrated by Li and Jensen [29], and the sequence has been implemented in a consumer level tablet by Hemmsen et al. [30].

In this paper, a 2-D VFI method is proposed for a portable ultrasound system combining SASB and DTO. The paper is an extension of [31]. B-mode and flow emissions are interleaved to obtain continuous data acquisition, and six emissions are used for each flow HRI. The TO is created in the HRIs due to the spatial distribution of the VSs, which are divided in two virtual apertures (VAs) separated by a lateral distance. The velocity estimation is, therefore, performed by correlating a number of HRIs in the two directions.

The remainder of the paper is organized as follows: the creation of the TO using SASB and the velocity estimator are introduced in Section II. The imaging setup and the processing are described in Section III. The experimental investigations are introduced in Section IV and the results for the constant flow measurements and in vivo images from a common carotid artery are presented in Section V. The results are finally discussed in Section VI.

\section{THEORY}

To estimate the 2-D velocity components, a point spread function (PSF) oscillating in both the axial and lateral directions is needed. For the analytical derivation of the PSF, readers are referred to [16] and [19]. The frequency of the lateral oscillation is $f_{x}=D p / 2 \lambda z$ in the case of a broad transmit beam. Here, $D$ is the lateral distance between the apertures in number of transducer elements, $p$ the pitch of the array, $\lambda$ the axial wavelength equal to $c / f_{0}$, and $z$ the distance from the apertures. If separated apertures are used in both transmit and receive, as for the method proposed here, the lateral frequency is doubled, and it is

$$
f_{x}=\frac{1}{\lambda_{x}}=\frac{D p}{\lambda z},
$$

where $\lambda_{x}$ is the lateral wavelength.

The expression in (1) is strictly valid only for continuous wave excitation and at the transmit focus, hence it represents an approximation for pulsed wave emissions. Inaccurate frequencies yield biased velocities, and for this reason, $f_{x}$ has to be directly estimated from the acquired ultrasound data using DTO, as previously shown in [21]. Also, DTO does not require the calibration of the beamformers at each depth as in conventional TO.

\section{A. TO creation using $S A S B$}

The novelty of the method proposed in this paper is the creation of the TO based on SASB using fixed-focused emissions in transmit and receive. This allows for a reduction of the data throughput that makes the method suitable for implementation on a wireless system. In this section, the acquisition and beamforming are described to provide an understanding of the method.

1) Acquisition: The acquisition sequence is schematically displayed in Fig. 1. $K$ emissions are sequentially transmitted at regular intervals of $T_{P R F}$, with $K=4$ in the figure. At each emission, $L$ transducer elements are excited and focused in a point located behind the array, therefore a diverging wave is emitted to insonify the area inside the dashed line. The focal positions can be considered VSs [26]-[28] and are laterally divided in two VAs to create a TO as described in the beamforming section. The VAs are laterally separated by a distance of $D$ transducer elements. In Fig. 1, the left VA is depicted in blue and the right VA in red.

2) Beamforming: The dual-stage beamforming involved in the method presented in this paper is shown in Fig. 2. For the sake of simplicity, a case is considered with one single VS per aperture $(K=2)$. The received data from $L$ transducers at each emission is focused by a first beamformer in a static point corresponding to the VS, and a LRL is obtained. The VS is therefore also considered a virtual receiver (VR), the response of which is the LRL. This is optimally focused only at the focal position. The first beamformer can be integrated in the probe handle [24], [25] and one single LRL has to be transmitted to the second stage after each emission. The data throughput between the probe and the processing unit is thus reduced by a factor $L$ compared to a full SA system. In the second stage, the data in the LRLs are refocused based on the VS/VR assumption. In Fig. 2, the PSF is shown in the secondstage boxes for the left VS on the top and for the right VS on the bottom. The computational complexity of the second stage equals that of a monostatic SA beamformer, where the LRLs are used in place of the transducer signals. For a thorough understanding of the second beamformer, readers are referred to [23].

A PSF oscillating in both directions is obtained in the HRI by coherently combining the responses from the VSs in the left and right VAs. The example of a double-oscillating PSF at a depth of $20 \mathrm{~mm}$ with a distance $D=48$ between the VAs is displayed in Fig. 2 after the summation. The top plot shows the 2-D PSF and the bottom plot the lateral sampling along the blue dashed line.

The shape of the PSF is affected by the distance $D$ as given by (1) and by the number $K$ of VSs. In particular, the lateral width of the PSF can be reduced by increasing $K$ to yield better resolution. On the other hand, a narrower PSF gives a broader lateral bandwidth, which in turn increases the lateral bias as discussed in [32]. Furthermore, the velocity range decreases with increasing $K$ due to aliasing as discussed in Sec. II-B 


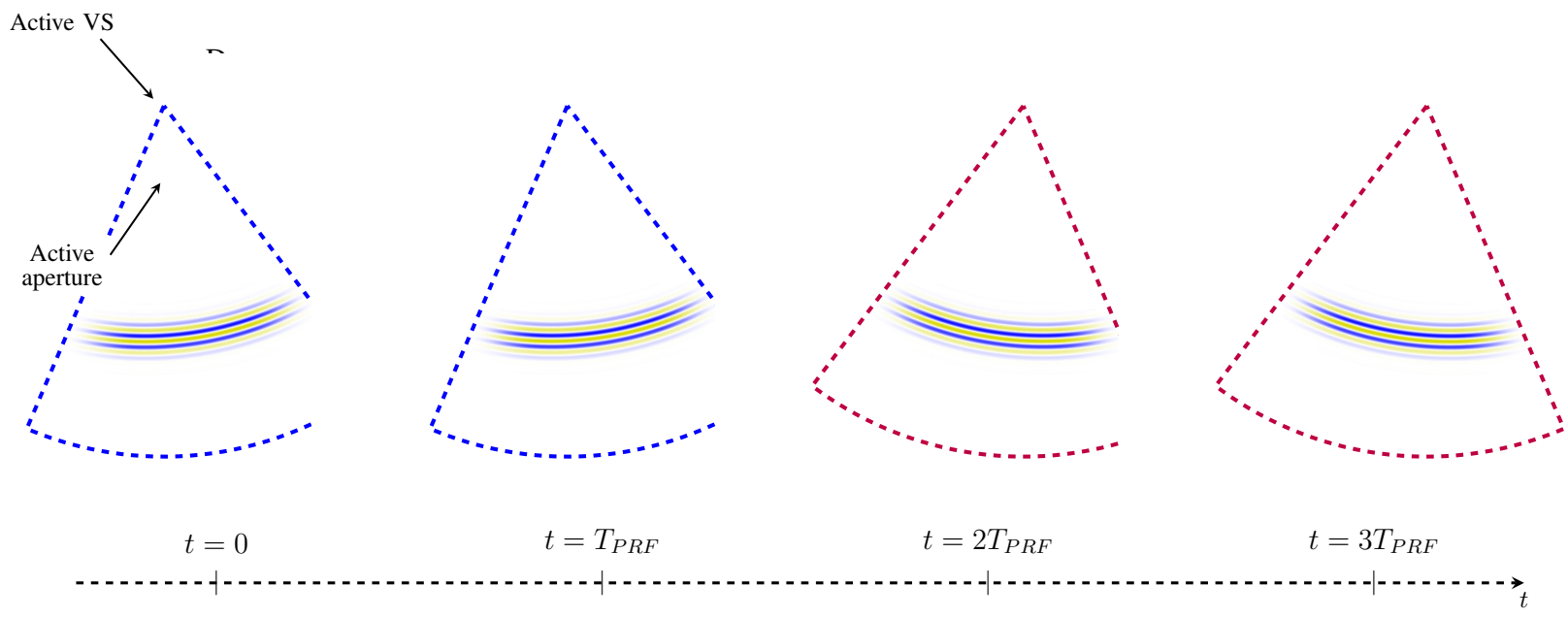

Fig. 1. Acquisition sequence for the SASB TO method. $K$ emissions are sequentially transmitted at regular intervals of $T_{P R F}$, with $K=4$ in this example. Each emission is focused behind the array, therefore a diverging wave is emitted to insonify the area inside the dashed line. The focal positions are considered VSs and are laterally divided in two VAs. The left VA is depicted in blue and the right one in red.

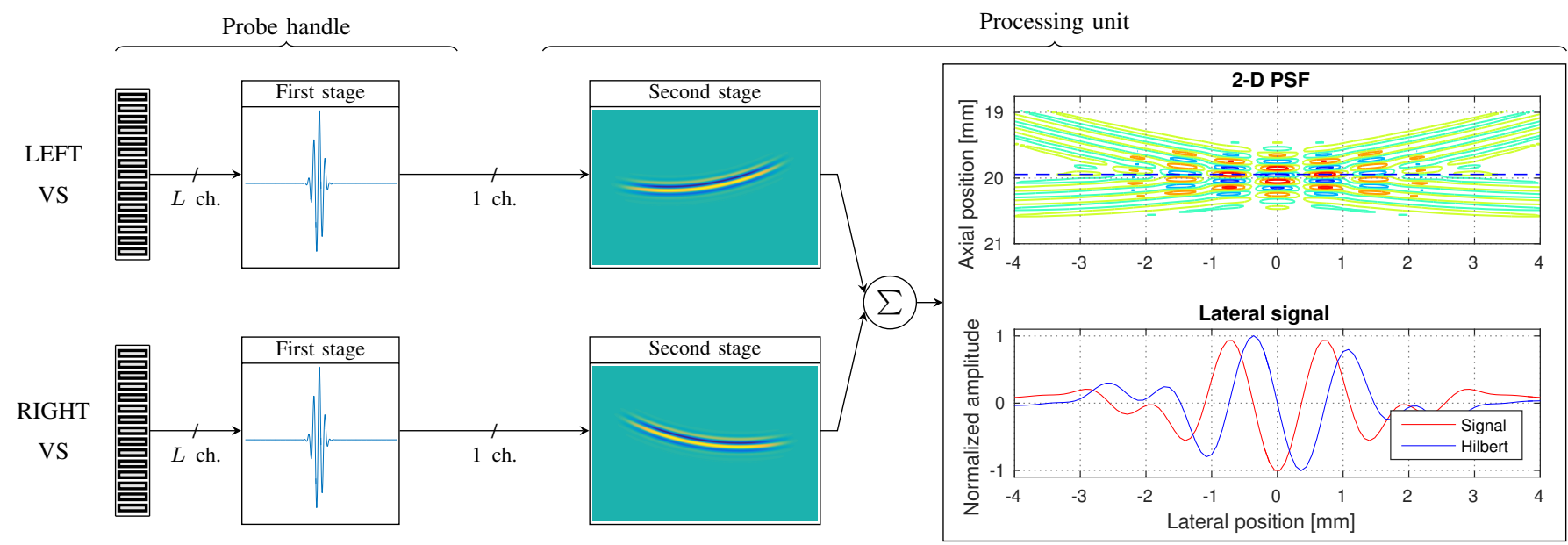

Fig. 2. Beamforming involved in the creation of a TO using SASB in the case with one single VS per aperture $(K=2)$. For each emission, the data received from $L$ transducers is focused by a first beamformer in a static point corresponding to the VS, and a LRL line is obtained. The VS is therefore also considered a virtual receiver (VR), the response of which is the LRL. In the second stage, the data in the LRLs are refocused based on the VS/VR assumption. The PSF is shown in the second-stage boxes for the left and right VSs. To obtain a double-oscillating PSF, the responses from the left and right VAs are coherently combined. The 2-D PSF is shown after summation in the top plot, and the lateral signal along the blue dashed line is shown in the bottom plot.

\section{B. Velocity estimation}

The velocity vectors can be estimated from the HRIs produced by the second-stage beamformer based on DTO [21]. The estimator's equations are reviewed here. To estimate the velocity at a given point, $M$ samples are selected in the HRI centred around the velocity point to create a lateral signal $s(m, n, e)$. Here, $m$ is the sample index along the lateral direction, i.e. $m=-M / 2, \ldots, M / 2-1, n$ is the axial sample index, and $e$ the index of the HRI. The analytic signal is calculated as

$$
r_{s q}(m, n, e)=s(m, n, e)+j \mathcal{H}_{m}\{s\}(m, n, e),
$$

where $\mathcal{H}_{m}$ is the Hilbert transform in the lateral direction $m$. The lateral frequency can be estimated at each depth as

$$
f_{x}(n, e)=\frac{1}{\lambda_{x}(n, e)}=\frac{\sum_{f=-F / 2}^{F / 2-1} \frac{f}{F \Delta x}\left|R_{s q}(f, n, e)\right|^{2}}{\sum_{f=-F / 2}^{F / 2-1}\left|R_{s q}(f, n, e)\right|^{2}},
$$

with $R_{s q}(f, n, e)=\mathcal{F}_{m}\left\{r_{s q}(m, n, e)\right\}$ the Fourier transform of $r_{s q}$ along the lateral direction $m, f$ the sample index in the discrete Fourier domain, $F$ the number of Fourier coefficients, and $\Delta x$ the lateral sampling period. The lateral frequencies from a number of HRIs can be averaged to obtain smoother estimates. In addition, $f_{x}$ can be averaged along the depth over a pulse length.

In the PSF in Fig. 2, a lateral beamformed signal and its Hilbert transform are displayed in the bottom graph from a point scatterer at a depth of $20 \mathrm{~mm}$ for $K=6$ and $D=48$. The analytic signal is used to calculate the lateral frequency using (3).

Two signals are created from $r_{s q}$,

$$
\begin{aligned}
& r_{1}(m, n, e)=r_{s q}(m, n, e)+j \mathcal{H}_{n}\left\{r_{s q}\right\}(m, n, e), \\
& r_{2}(m, n, e)=r_{s q}(m, n, e)-j \mathcal{H}_{n}\left\{r_{s q}\right\}(m, n, e),
\end{aligned}
$$

where $\mathcal{H}_{n}$ is the Hilbert transform in the axial direction $n$, 
and the lag-1 autocorrelation functions are calculated as

$$
\begin{aligned}
& R_{1}(1)=\frac{1}{M(N-1)} \sum_{m=-M / 2}^{M / 2-1} \sum_{e=0}^{N-2} r_{1}^{*}(m, n, e) r_{1}(m, n, e+1), \\
& R_{2}(1)=\frac{1}{M(N-1)} \sum_{m=-M / 2}^{M / 2-1} \sum_{e=0}^{N-2} r_{2}^{*}(m, n, e) r_{2}(m, n, e+1) .
\end{aligned}
$$

The autocorrelation estimates in (5) are averaged over $M$ lateral samples and $N$ HRIs. The lateral and axial velocities are finally estimated as [18], [21]

$$
\begin{aligned}
v_{x}= & \frac{\lambda_{x}}{2 \pi 2 T} \times \\
& \arctan \left(\frac{\Im\left\{R_{1}(1)\right\} \Re\left\{R_{2}(1)\right\}+\Im\left\{R_{2}(1)\right\} \Re\left\{R_{1}(1)\right\}}{\Re\left\{R_{1}(1)\right\} \Re\left\{R_{2}(1)\right\}-\Im\left\{R_{1}(1)\right\} \Im\left\{R_{2}(1)\right\}}\right), \\
v_{z}= & \frac{\lambda}{2 \pi 4 T} \times \\
& \arctan \left(\frac{\Im\left\{R_{1}(1)\right\} \Re\left\{R_{2}(1)\right\}-\Im\left\{R_{2}(1)\right\} \Re\left\{R_{1}(1)\right\}}{\Re\left\{R_{1}(1)\right\} \Re\left\{R_{2}(1)\right\}+\Im\left\{R_{1}(1)\right\} \Im\left\{R_{2}(1)\right\}}\right),
\end{aligned}
$$

with $T$ the period between successive HRIs, and $\Re$ and $\Im$ the real and imaginary parts. The velocities $v_{x}$ and $v_{z}$ can be estimated for every point in the image after $N$ HRIs are acquired.

The value of $v_{x}$ in (6) is directly proportional to $\lambda_{x}$, therefore it is important to use the estimated value and avoid biasing of the lateral velocities. The length $M$ used in (3) can, in general, be different than that used in (5). Longer signals in (3) provide better estimates due to the reduced spectral broadening, while lower SD can be achieved by averaging (5) over a greater $M$. Nevertheless, this reduces the spatial resolution of the estimates. The distance $D$ can be chosen to optimize the lateral wavelength as pointed out in (1).

The range of detectable velocities is limited by aliasing, which is more severe here due to the acquisition of the HRIs over a number of $K$ emissions. The limit for $v_{x}$ is

$$
\left|v_{x}\right| \leq v_{x}^{\max }=\frac{1}{4} \frac{\lambda_{x}}{T}=\frac{1}{4} \frac{\lambda z}{D p} \frac{1}{T},
$$

where (1) is used for $\lambda_{x}$, and for $v_{z}$ it is

$$
\left|v_{z}\right| \leq v_{z}^{\max }=\frac{1}{8} \frac{\lambda}{T}
$$

The maximum $v_{x}$ is lower for increased $D$ due to the shorter lateral wavelength. Both (8) and (9) are inversely proportional to $T$, and this is in turn given by the $T_{P R F}$ and the number $K$ of VSs. Therefore, a tradeoff exists between the resolution of the PSF and the maximum detectable velocity.

\section{METHODS}

The method was first investigated with constant flow measurements in a flow rig system, and then the measurement of a common carotid artery was performed to test the performance in vivo. A parametric study was carried out to optimize the imaging parameters prior to the in vivo measurement. In this section, the details are provided about the imaging setup and the processing parameters used in the experiments.
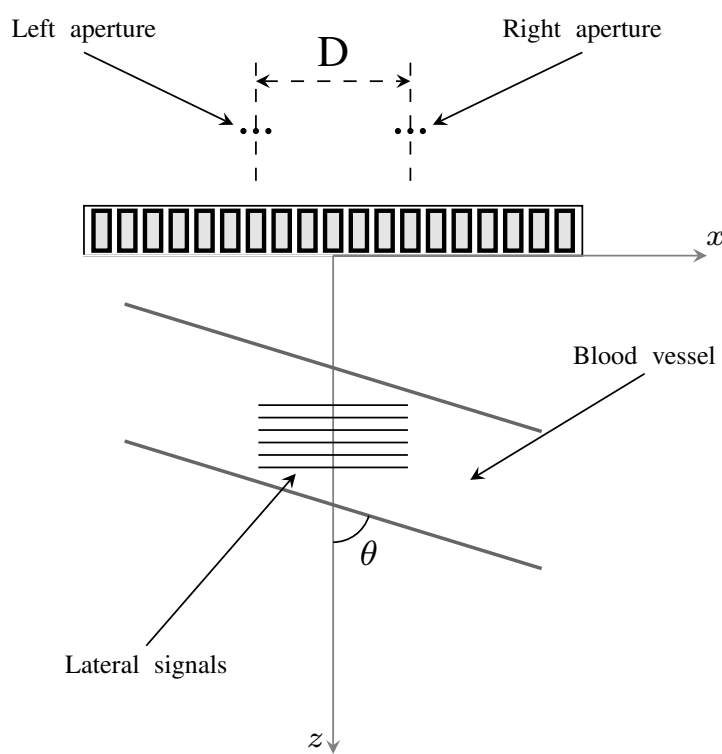

Fig. 3. Imaging setup including the transducer array and the imaged vessel. The vessel was positioned below with a beam-to-flow angle $\theta . K=6 \mathrm{VSs}$ were used for each flow HRI and were spatially divided in two VAs separated by a distance $D$.

TABLE I

TRANSDUCER PARAMETERS

\begin{tabular}{l|c|c}
\hline Parameter & Value & Unit \\
\hline Array type & Linear & - \\
Element pitch - $p$ & 0.2 & $\mathrm{~mm}$ \\
Element height & 6 & $\mathrm{~mm}$ \\
Number of elements & 192 & - \\
Elevation focus & 38 & $\mathrm{~mm}$ \\
Center frequency & 4.1 & $\mathrm{MHz}$ \\
\hline
\end{tabular}

\section{A. Imaging setup}

In Fig. 3, the setup is shown including the transducer array and the imaged vessel. The position of the VAs relative to the array is also displayed. A $0.55 \lambda$-pitch linear array was used with the parameters shown in Table I. The array was connected to the SARUS scanner [33] for the acquisition of the element data. The vessel was positioned below the transducer with a beam-to-flow angle $\theta$. A duplex sequence was designed with the parameters in Table II. The VAs were positioned behind the transducer and steered toward the center of the image with an angle of $\pm 2.5^{\circ}$. The distance $D$ was optimized in the parametric study described in Section IV, and its values are reported in Table III, where the default is displayed in bold. The B-mode VSs were regularly distributed between $\pm 15 \mathrm{~mm}$ in the lateral direction.

The pulse repetition frequency was set to $P R F=9 \mathrm{kHz}$ for the constant flow and $15 \mathrm{kHz}$ for the in vivo measurements. B-mode and flow emissions were interleaved to achieve continuous data acquisition, i.e. one B-mode pulse was transmitted after every $K=6$ flow emissions, with an effective repetition frequency $P R F_{\text {eff }}=P R F /(K+1)$. The period $T$ in (6), (7), (8), and (9) is, thus, equal to $1 / P R F_{\text {eff }}=(K+1) / P R F$. 
TABLE II

FIXED IMAGING SETUP AND PROCESSING PARAMETERS

\begin{tabular}{|c|c|c|c|}
\hline Parameter & Flow & B-mode & Value \\
\hline Imaging setup & & & \\
\hline Excitation & 4 cycles & 2 cycles & - \\
\hline Center frequency $-f_{0}$ & \multicolumn{2}{|c|}{4.1} & $\mathrm{MHz}$ \\
\hline Transmit apodization & \multicolumn{2}{|c|}{ Tukey $(\alpha=0.6)$} & - \\
\hline Receive apodization & \multicolumn{2}{|c|}{ Tukey $(\alpha=0.6)$} & - \\
\hline VS axial position & -15 & -30 & $\mathrm{~mm}$ \\
\hline Active elements & 64 & 96 & - \\
\hline f-number - f\# & -1.17 & -1.56 & - \\
\hline Number of VSs & $K=6$ & 64 & - \\
\hline VA apodization & Rect & - & - \\
\hline Pulse repetition freq. $-P R$ & & & \\
\hline Constant flow & \multicolumn{2}{|c|}{9} & $\mathrm{kHz}$ \\
\hline In-vivo & \multicolumn{2}{|c|}{15} & $\mathrm{kHz}$ \\
\hline Processing & & \\
\hline Lateral sampling interval & \multirow{2}{*}{\multicolumn{2}{|c|}{0.1}} & $\mathrm{~mm}$ \\
\hline Axial sampling frequency & & 35 & $\mathrm{MHz}$ \\
\hline
\end{tabular}

TABLE III

VARIED IMAGING SETUP AND PROCESSING PARAMETERS

\begin{tabular}{l|c|c}
\hline Parameter & Value & Unit \\
\hline Distance between VAs - $D$ & $16-24-32-40$ & transducer \\
& $\mathbf{4 8 - 5 6 - 6 4}$ & elements \\
& $4-8-16$ & \\
Lateral signal length - $M$ & $24-\mathbf{3 2}-48$ & samples \\
& $4-8-16-\mathbf{3 2}$ & \\
Number of HRIs - $N$ & $64-96-128$ & - \\
\hline
\end{tabular}

\section{B. Processing}

The processing scripts were developed in MATLAB (The MathWorks, Inc., Natick, MA, USA), and the beamformation was performed using the BFT3 toolbox [34]. The measured element data were beamformed in the first-stage beamformer and then matched filtered. For the flow data, a stationary echo cancelling filter was used as described in Section III-C. The HRIs were beamformed by the second stage in a Cartesian grid and were Hilbert transformed in the lateral direction. The directional signals were created by selecting the lateral samples. The frequency $f_{x}$ was estimated as in (3) for all the depths using signals of $M=64$ samples, and averaged over $N=64$ HRIs and a pulse length in the axial direction. The curve $f_{x}$ was then inverted, and a line was fitted to the $\lambda_{x}$. The estimation of $f_{x}(n, e)$ was performed only once, and can be considered an initialization procedure. The lateral frequency displayed in Fig. 4 was estimated from a speckle phantom measurement with $D=48$. The hyperbole displayed in black was obtained from the linear fit to the estimated $\lambda_{x}$. The green curve shows the theoretical $f_{x}$ calculated using (1), with $c=1540 \mathrm{~m} \mathrm{~s}^{-1}$ and $z$ the axial distance from the VAs. The theoretical equation overestimates $f_{x}$ and would provide biased velocities.

The length $M$ used for the velocity estimation was changed as in Table III, where the default value is displayed in bold. The autocorrelation functions in (5) were calculated from $N$ consecutive HRIs and averaged over $M$ lateral samples and a pulse length in the axial direction. The performance with varying $N$ was investigated in the parametric study, and the values are reported in Table III with the default value displayed

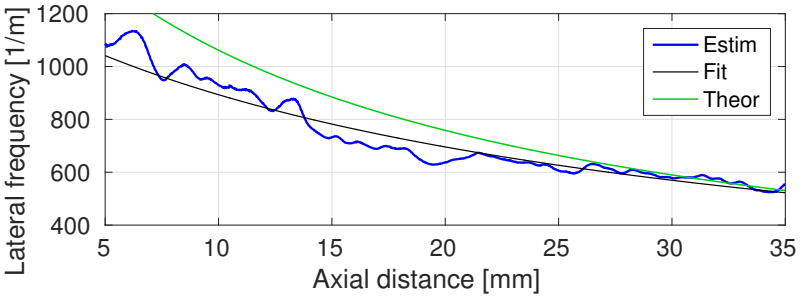

Fig. 4. Lateral frequency estimated from a speckle phantom measurement with $D=48$ using lateral signals of $M=64$ samples. The estimated frequencies were averaged over $N=64 \mathrm{HRIs}$ and a pulse length in the axial direction. The hyperbole displayed in black was obtained from the linear fit to the estimated $\lambda_{x}$. The green curve shows the theoretical frequency calculated using (1), with $c=1540 \mathrm{~m} \mathrm{~s}^{-1}$.

in bold. The velocity estimates were averaged over a pulse length.

The B-mode HRIs were beamformed by the second stage beamformer and the analytic signals were calculated using a Hilbert transform in the axial direction. The envelope-detected and log-compressed images were shown with a dynamic range of $40 \mathrm{~dB}$. The processing parameters for the flow and B-mode data are reported in Table II.

\section{Clutter filter}

A schematic representation of the amplitude spectrum of the received signal $G(f)$ is displayed in Fig. 5 to show the principle of operation of the clutter filter used in this study. The tissue component $G_{t}(f)$ is low-frequency, and its bandwidth depends upon the velocity distribution in the tissue. $G_{b}(f)$ is the signal from the blood and $G_{n}(f)$ is electronic noise. The energy of the signals is represented by the coloured areas. The goal of the clutter filter is to minimize the energy of $G_{t}$ while preserving that of $G_{b}$.

A dual-stage filter was used. The first stage is a high-pass moving-average-subtraction filter $H_{h p}(f)$ with a $-3 \mathrm{~dB}$ cutoff frequency of $120 \mathrm{~Hz}$ used on the LRLs. In ideal conditions of stationary tissue, $G_{t}(f)$ is narrowband and is effectively attenuated by $H_{h p}(f)$. However, significant components leak in the blood spectrum when tissue velocities cause a broadening of $G_{t}(f)$. In general, these components have high amplitude and disrupt the velocity estimation.

The second stage consists of an amplitude threshold $E$ and sets $G(f)=E \forall f: G(f)>E$. This filter is used on the HRIs and removes any spectral components with amplitudes above the threshold $E$. As a result, the energy of $G_{t}$ is further attenuated compared with that of $G_{b}$. The threshold $E$ was determined after inspecting the spectrum of the signal received from inside the vessel calculated by means of a windowed fast Fourier transform (FFT). The time-domain signals were then restored after filtering by performing an inverse FFT. The approach has been previously used and described in [36] and [37].

The energy-based filter was used in addition to the moving average as the latter alone failed to properly attenuate the signal from the tissue during the systolic phase. However, the choice of $E$ is critical to the performance of the estimator. If 


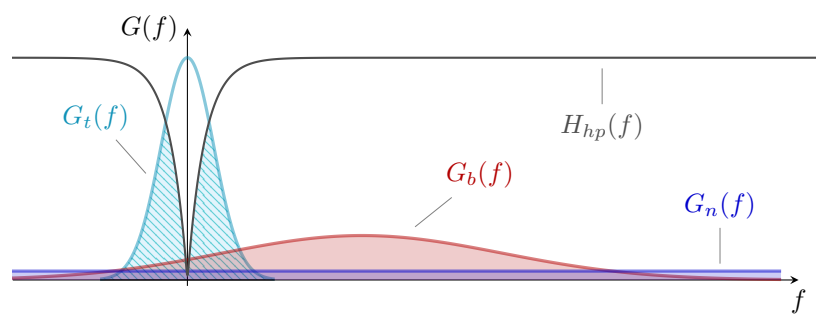

(a)

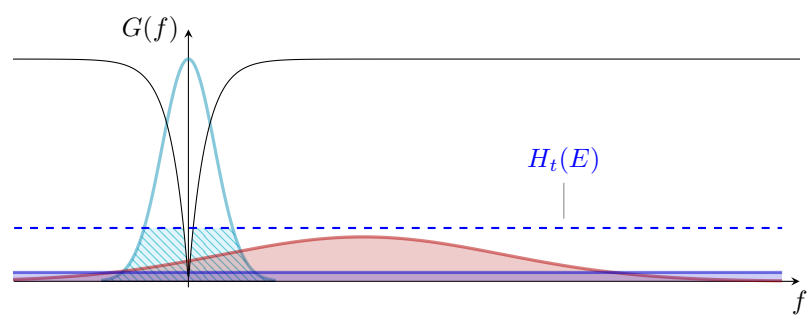

(b)

Fig. 5. Schematic representation of the amplitude spectrum of the received signal $G(f) . G_{t}(f)$ is the spectrum of the signal scattered by the surrounding tissue; $G_{b}(f)$ is the spectrum of the signal from the blood; and $G_{n}(f)$ is white noise from the measuring system. The energy of the components is highlighted by the coloured areas. (a): A high-pass filter $H_{h p}(f)$ is used to reduce the energy of $G_{t}$ (area under the curve); (b): a second stage $H_{t}(E)$ is used to remove any spectral components with amplitude greater than a threshold $E$.

E is higher than the amplitude of the blood signal, a higher residual energy from the tissue signal will bias the estimation of the blood velocity. On the other hand, a low threshold will filter out energy from the blood spectrum.

\section{EXPERIMENTAL INVESTIGATIONS}

\section{A. Constant flow measurements}

Measurements were performed on a flow rig system consisting of a centrifugal pump (Cole-Parmer, Vernon-Hills, IL, USA) circulating a blood mimicking fluid and a vessel with a radius of $6 \mathrm{~mm}$ immersed in a water bath. The entrance length of the vessel ensured a fully developed parabolic, laminar flow profile. The volume flow was measured for reference by a magnetic flow meter (MAG3000, Danfoss, Nordborg, Denmark) and was set to obtain a peak velocity of approximately $0.2 \mathrm{~m} \mathrm{~s}^{-1}$. The relative bias and SD were calculated inside the vessel from 50 independent velocity profiles.

A parametric study was first carried out to investigate the performance of the method as a function of the distance $D$, the length $M$, and the number $N$ of HRIs used for the velocity estimation. The vessel was placed at a depth of approximately $20 \mathrm{~mm}$ with a beam-to-flow angle $\theta=75^{\circ}$. This setup was chosen to optimize the imaging parameters for the measurement of a common carotid artery.

The constant parabolic flow profile with $\theta=75^{\circ}$ was in addition measured with the default setup $(D=48 ; M=$ $32 ; N=32$ ) at a depth of approximately $30 \mathrm{~mm}$ and $40 \mathrm{~mm}$, and at $20 \mathrm{~mm}$ for flow angles of $90^{\circ}$ and $65^{\circ}$.

The range in (8) was shifted to $\left[-\frac{1}{4} v_{x}^{\max }, \frac{7}{4} v_{x}^{\max }\right]$ to match the velocities expected in the in vivo measurement. At the depth of $20 \mathrm{~mm}$ with $P R F=9 \mathrm{kHz}, v_{x}^{\max }=0.44 \mathrm{~m} \mathrm{~s}^{-1}$ and the velocity ranges are

$$
\begin{aligned}
& -0.11 \mathrm{~m} \mathrm{~s}^{-1} \leq v_{x} \leq 0.77 \mathrm{~m} \mathrm{~s}^{-1} \\
& -0.12 \mathrm{~m} \mathrm{~s}^{-1} \leq v_{z} \leq 0.12 \mathrm{~m} \mathrm{~s}^{-1} .
\end{aligned}
$$

For the vessel at $65^{\circ}$, an axial velocity $v_{z}=v_{z}^{\max }=0.12 \mathrm{~m} \mathrm{~s}^{-1}$ gives a maximum peak velocity equal to $0.28 \mathrm{~m} \mathrm{~s}^{-1}$. A peak velocity of $0.2 \mathrm{~m} \mathrm{~s}^{-1}$ was used to avoid aliasing.

To show the performance of the method with lateral velocities closer to those detected in vivo, the flow rig measurements were repeated with the default setup and a vessel positioned at $90^{\circ}$. The peak velocity was set to $0.32,0.44$, and $0.5 \mathrm{~m} \mathrm{~s}^{-1}$ with $P R F=9 \mathrm{kHz}$. The velocity was then kept equal to $0.5 \mathrm{~m} \mathrm{~s}^{-1}$ to maintain laminar flow, and the $P R F$ was lowered to 7.5 and $6.4 \mathrm{kHz}$ to emulate velocities of 0.6 and $0.7 \mathrm{~m} \mathrm{~s}^{-1}$, respectively.

\section{B. Real-time tablet implementation}

The proposed method was implemented on a consumer level tablet for a proof-of-concept investigation of the feasibility of the wireless data transmission and real-time processing. The LRLs beamformed by the first-stage from the flow rig acquisition at $90^{\circ}$ and $0.2 \mathrm{~m} \mathrm{~s}^{-1}$ were sampled at $20 \mathrm{MHz}$ and sent to a Nexus 9 (HTC Corp., Taoyuan, Taiwan) through an RT-AC68U (ASUS, Taipei, Taiwan) Wi-Fi router to emulate a wireless probe. The tablet integrates a dual-core 64-bit CPU at $2.3 \mathrm{GHz}$ and a Tegra K1 GPU, NVIDIA Corp., Santa Clara, CA, USA, with 192 shader cores and 2 GB DDR3 RAM. The LRLs were buffered into the tablet's memory and then beamformed by the second-stage. Sixteen HRIs of $32 \times 512$ samples were used for each velocity estimation. B-mode and VFI processing, including beamforming, stationary echo cancelling, velocity estimation and displaying, were implemented on the tablet's GPU through the OpenGL ES 3.1 API. The 2-D velocities were estimated from 16 HRIs in a region of $0.8 \mathrm{~cm} \times 1.9 \mathrm{~cm}$. The processing and wireless transmission were performed simultaneously to take into account the contribution of the Wi-Fi chipset to the heating of the system.

\section{In vivo measurement}

An in vivo measurement was performed after approval by the Danish National Committee on Biomedical Research Ethics. The right common carotid artery of a healthy 28year-old volunteer was scanned with a longitudinal view by an experienced radiologist using the setup in Section III-A. The volunteer was asked to rest in a supine position for approximately $10 \mathrm{~min}$ before the measurement to ensure steady flow conditions.

The spatial-peak temporal-average intensity and mechanical index (MI) of the sequence were measured prior to the in vivo experiment to verify the compliance of the FDA safety guidelines. These require $\mathrm{MI} \leq 1.9$ and $I_{\text {spta }} \leq 720 \mathrm{~mW} \mathrm{~cm}^{-2}$ [38]. An Acoustic Intensity Measurement System AIMS III (Onda Corp., Sunnyvale, CA, USA) was used with a hydrophone Onda HGL-0400 following the procedure in [39]. The PRF was set to $500 \mathrm{~Hz}$ during the measurement to 

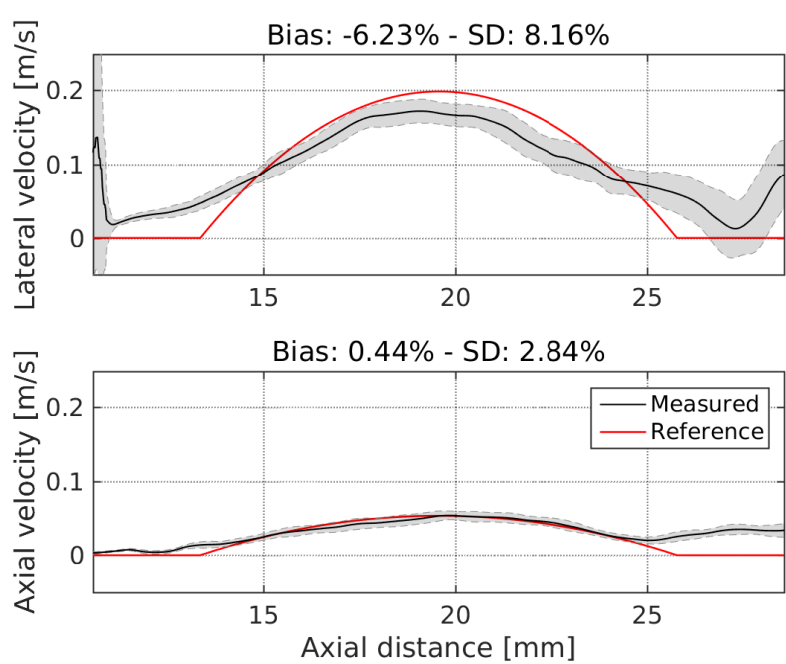

Fig. 6. Measured and reference profiles for the lateral (top) and axial (bottom) velocities measured in the flow rig for the default setup: $D=48 ; M=32$; $N=32$. The vessel was placed at a depth of $20 \mathrm{~mm}$ with a flow angle of $75^{\circ}$.

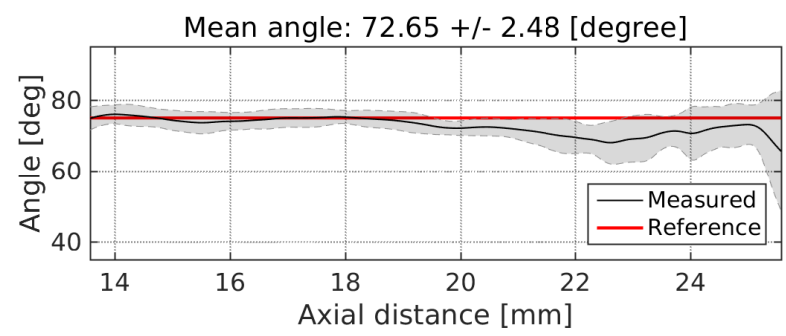

Fig. 7. Estimated angle inside the boundaries of a vessel measured for the default setup: $D=48 ; M=32 ; N=32$. The vessel was placed at a depth of $20 \mathrm{~mm}$ with a flow angle of $75^{\circ}$.

avoid reverberations in the water tank. The derated MI was equal to 0.91 and the derated $I_{\text {spta }}=10.19 \mathrm{~mW} \mathrm{~cm}^{-2}$. For $P R F=15 \mathrm{kHz}$, the intensity is therefore $305.74 \mathrm{~mW} \mathrm{~cm}^{-2}$, which satisfies the FDA regulations. The temperature was also measured at the transducer surface and was within the FDA limits.

A sequence of $9.5 \mathrm{~s}$ of data was saved, and the processing was carried out off-line as described in Section III-B. To match the non-stationary characteristic of the flow, 16 HRIs were used for each flow estimation. The velocity estimates were shown with a frame rate of 350 frames per second (FPS), while the frame rate of the B-mode sequence was 33 FPS. The VFI video was paced down by a factor 14. A binary mask was generated from the B-mode images to discriminate the vessel area from the surrounding tissue. The velocity estimates were filtered using a median filter in a temporal window of $25 \mathrm{~ms}$ and a spatial window of $1 \times 1 \mathrm{~mm}^{2}$.

\section{RESULTS}

\section{A. Constant flow measurement}

The estimated velocities for the default setup - $D=48$; $M=32 ; N=32$ - are shown in Fig. 6 for the vessel at $20 \mathrm{~mm}$ and $75^{\circ}$. The mean profiles (black) and the SDs
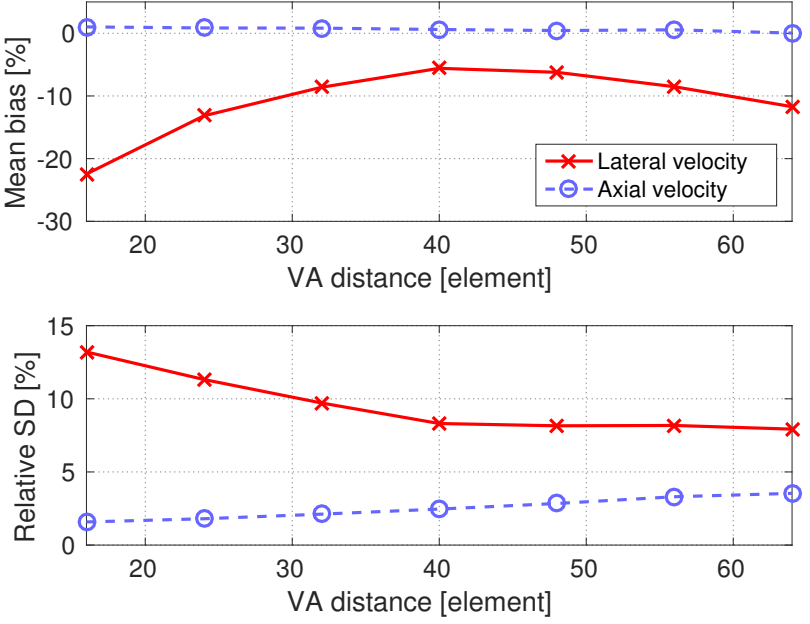

Fig. 8. Mean bias (top) and SD (bottom) as a function of the distance $D$ between the VAs for the lateral and axial velocities. These were measured in the flow rig at a depth of $20 \mathrm{~mm}$ with a flow angle of $75^{\circ}$.
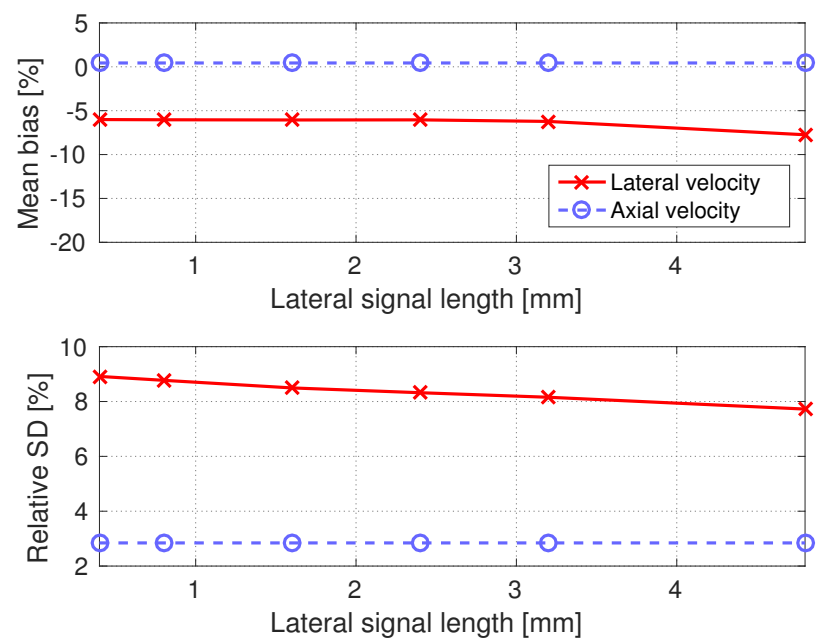

Fig. 9. Mean bias (top) and SD (bottom) as a function of the length $M$ of the lateral signal expressed in $\mathrm{mm}$. Lateral and axial flow profiles were measured in the flow rig at a depth of $20 \mathrm{~mm}$ with a flow angle of $75^{\circ}$.

(shaded regions) are plotted along with the reference profiles (red) calculated from the measured volume flow. The lateral and axial velocities are displayed in the top and bottom graphs, respectively. The mean bias is $-6.23 \%$ and $0.44 \%$ in the lateral and axial directions, respectively. The mean SD is $8.16 \%$ and $2.84 \%$. In Fig. 7, the estimated angle is shown as a function of the depth for the points inside the vessel boundaries, with a mean value of $72.65 \pm 2.48^{\circ}$.

The results of the parametric study are displayed in Fig. 8, 9 , and 10. The mean bias (top graph) and SD (bottom graph) are shown in Fig. 8 as a function of $D$ for the lateral and axial profiles. By increasing $D$, shorter oscillation wavelengths are obtained in the lateral direction. For $D<40, \lambda_{x}$ is longer than the lateral signal $(M=32)$, and this results in increased bias of the lateral velocity. The mean lateral bias is between $-5.6 \%$ and $-11.7 \%$ for $D \geq 40$, and the SD is between $8.3 \%$ and $7.9 \%$. The axial bias is lower than $1 \%$ in all the cases and the SD is between $1 \%$ and $3 \%$. 

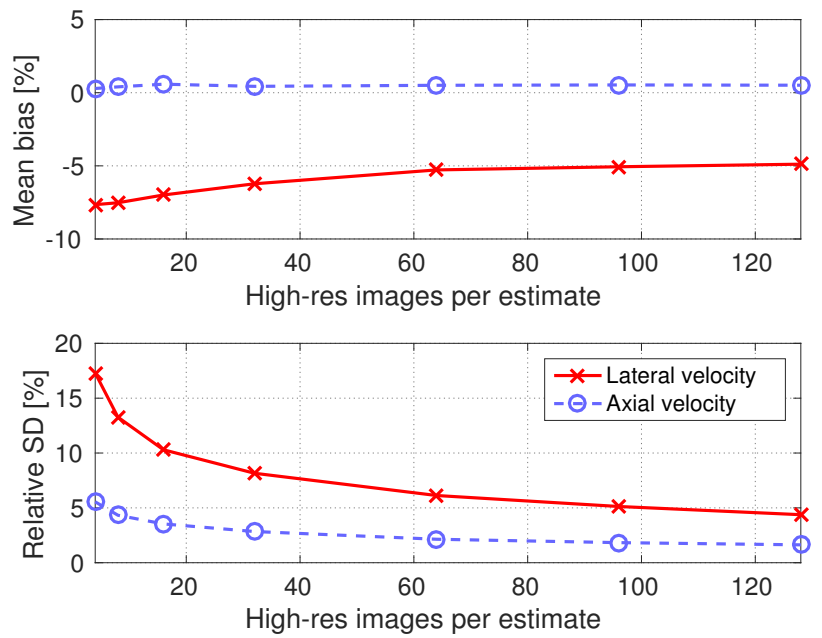

Fig. 10. Mean bias (top) and SD (bottom) as a function of the number $N$ of HRIs used per velocity estimate. Lateral and axial flow profiles were measured in the flow rig at a depth of $20 \mathrm{~mm}$ with a flow angle of $75^{\circ}$.
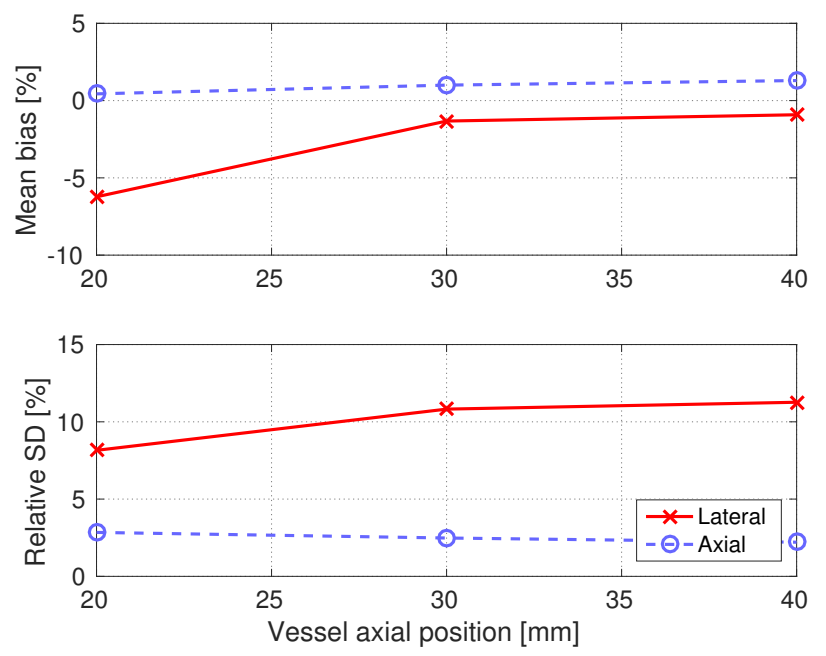

Fig. 11. Mean bias (top) and SD (bottom) for the lateral and axial flow profiles measured in the flow rig with a flow angle of $75^{\circ}$ at depth from 20 to $40 \mathrm{~mm}$.

In Fig. 9, a lower lateral SD is obtained by increasing $M$, while the other values are unaffected. Signals of only 4 samples can be used to reduce the computational complexity of the calculations. $M=32$ was used in the rest of the experiments.

The mean bias in Fig. 10 is only slightly affected by increasing $N$, while the SDs decrease significantly. For $N=128$, the $\mathrm{SD}$ is $4.4 \%$ and $1.6 \%$ in the lateral and axial directions.

In Fig. 11, the mean bias and SD are plotted as a function of the axial position of the vessel from 20 to $40 \mathrm{~mm}$ with $\theta$ $=75^{\circ}$. The lateral bias is between $-6.2 \%$ and $-0.9 \%$, while the SD is between $8.2 \%$ and $11.2 \%$. Constant performance is obtained for the axial velocity.

In Table IV, the results are show for the vessel at a depth of $20 \mathrm{~mm}$ with beam-to-flow angles of $90^{\circ}, 75^{\circ}$, and $65^{\circ}$.

Finally, the mean bias and SD with increasing velocity are shown in Fig. 12. For velocities up to $0.6 \mathrm{~m} \mathrm{~s}^{-1}$, the lateral bias is between $-7.4 \%$ and $2.1 \%$ and the SD between $6.1 \%$
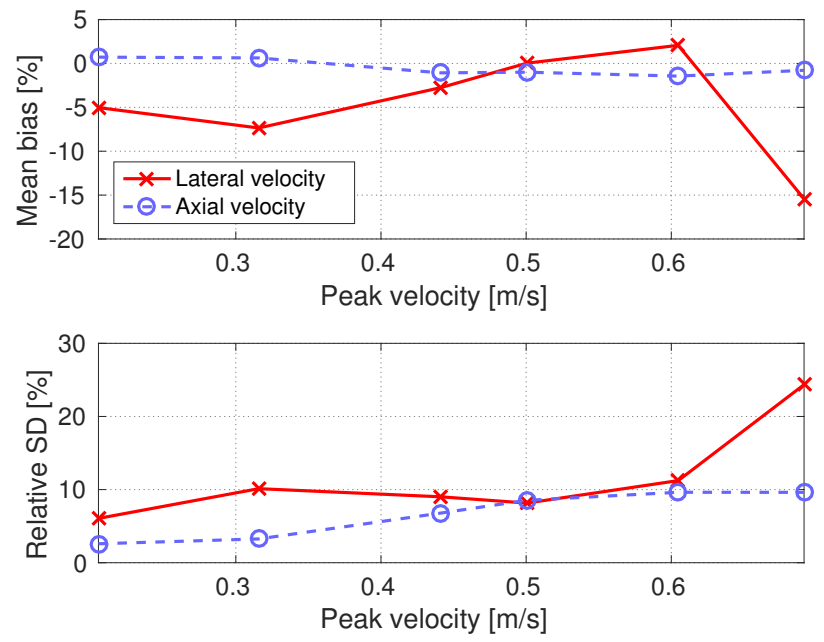

Fig. 12. Mean bias (top) and SD (bottom) for the lateral and axial flow profiles measured in the flow rig with a flow angle of $90^{\circ}$ at $20 \mathrm{~mm}$ for peak velocities between 0.2 and $0.7 \mathrm{~m} \mathrm{~s}^{-1}$. The velocities between 0.2 and $0.5 \mathrm{~m} \mathrm{~s}^{-1}$ were detected with PRF $=9 \mathrm{kHz}$, while for higher velocities the peak was kept equal to $0.5 \mathrm{~m} \mathrm{~s}^{-1}$ to avoid turbulent flow and the PRF was set to 7.5 and $6.4 \mathrm{kHz}$.

TABLE IV

CONSTANT FLOW MEASUREMENTS RESULTS

\begin{tabular}{l|cc|cc|c}
\hline Flow & \multicolumn{2}{|c|}{ Lateral } & \multicolumn{2}{c|}{ Axial } & Estimated Angle \\
angle & Bias & SD & Bias & SD & \\
\hline $90^{\circ}$ & $-5.03 \%$ & $6.06 \%$ & $0.72 \%$ & $2.59 \%$ & $89.13 \pm 0.79^{\circ}$ \\
$75^{\circ}$ & $-6.23 \%$ & $8.16 \%$ & $0.44 \%$ & $2.84 \%$ & $72.65 \pm 2.48^{\circ}$ \\
$65^{\circ}$ & $5.62 \%$ & $9.58 \%$ & $0.32 \%$ & $2.84 \%$ & $66.70 \pm 2.86^{\circ}$ \\
\hline
\end{tabular}

and $11.2 \%$. An increased bias and SD (-15.4\% and 24.3\%) are reported for $0.7 \mathrm{~m} \mathrm{~s}^{-1}$ because of the aliasing.

\section{B. Real-time tablet implementation}

The maximum data throughput between the tablet and the Wi-Fi router was first measured and was higher than $30 \mathrm{MB} / \mathrm{s}$. The peak frame rate of the tablet VFI processing was 27 FPS (37 ms/frame), which corresponds to a data rate of $14 \mathrm{MB} / \mathrm{s}$. The processing frame rate was gradually lowered due to the heating of the chipset and was 15 FPS after $12 \mathrm{~min}$ of continuous processing. It is, however, suitable for performing real-time imaging into the tablet. The data rate is lower than the maximum throughput between the tablet and the router, therefore the wireless transmission of the data can be achieved. The real time wireless transmission could not be tested concurrently with the acquisition of the data, since a wireless probe was not available.

\section{In vivo measurement}

The result of the in vivo measurement is shown in Fig. 13 and a video sequence is available in the digital version of the paper. In Fig. 13a and 13b, the B-mode and VFI images are shown at late diastole and peak systole, respectively. The arrows show the local velocity vectors and the underlying color map encodes the magnitude and angle as in the color wheel in the bottom-right corner. The velocity magnitude at the position indicated by the yellow circle in Fig. 13a is displayed in Fig. $13 \mathrm{c}$ for nine cardiac cycles. The red and magenta dots show 


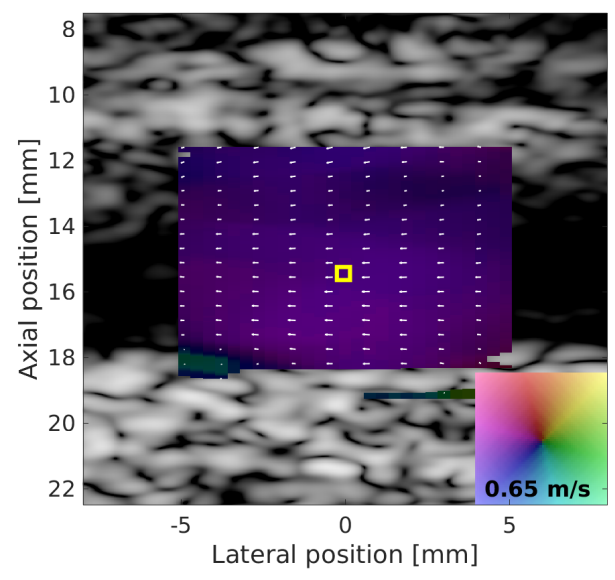

(a)

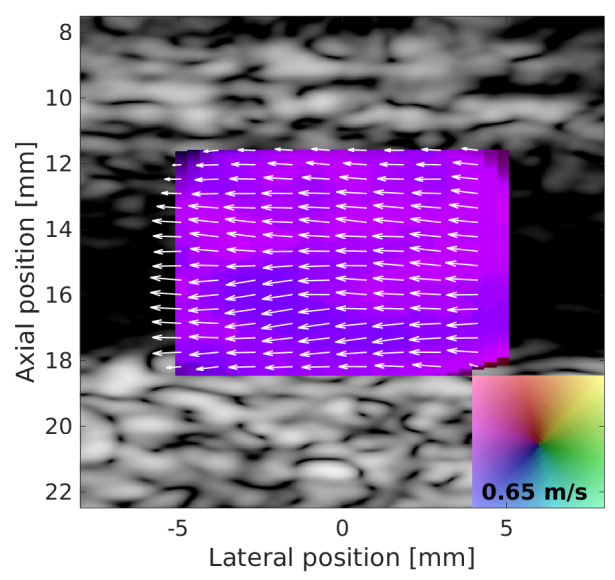

(b)

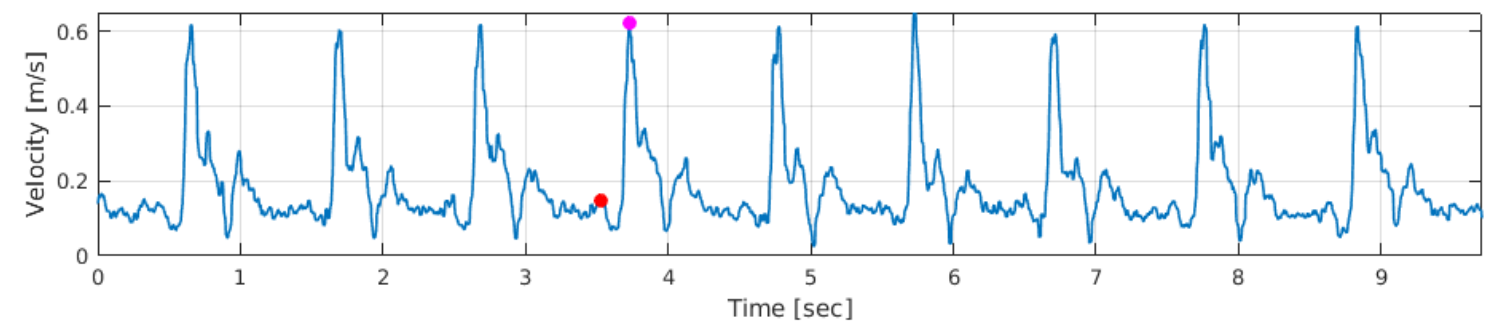

(c)

Fig. 13. VFI plot of a common carotid artery at late diastole (a) and peak systole (b) measured in vivo. In (c), the velocity magnitude at the position indicated by the yellow circle in (a) is displayed as a function of the time for the nine measured cardiac cycles. The red and magenta circles shows the times at which the VFI images are plotted. The mean PSV for the nine measured heart cycles is $0.62 \mathrm{~m} \mathrm{~s}^{-1}$ with a SD of $2.9 \%$.

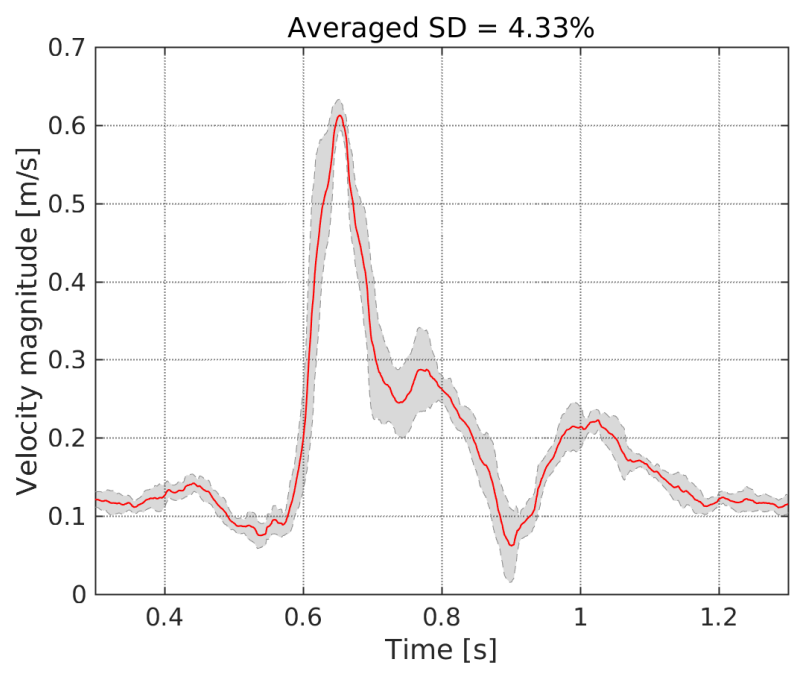

Fig. 14. Mean profile and relative SD calculated from 9 cardiac cycles measured in the common carotid artery. The relative SD averaged over a cardiac cycle is $4.33 \%$.

the times at which the velocities are plotted in the top figures. The mean PSV from the nine heart cycles is $0.62 \mathrm{~m} \mathrm{~s}^{-1}$ with a SD of $2.9 \%$.

The nine cycles in Fig. 13c were finally aligned by finding the peaks of the cross-correlation functions to calculate the mean profile and the relative SD plotted in Fig. 14. The relative
SD averaged over a cardiac cycle is $4.33 \%$. It should be noted that such measurements can be obtained anywhere in the VFI region at any time.

\section{Discussion}

A VFI method was presented for a wireless ultrasound system combining SASB and DTO. The performance of the method was investigated with constant flow measurements in a flow rig system. A lateral mean bias between $-5 \%$ and $6.2 \%$ was achieved, with a SD between $6 \%$ and $9.6 \%$ for beam-to-flow angles from $65^{\circ}$ to $90^{\circ}$. The axial mean bias was lower than $1 \%$ with a SD around $2 \%$. The scan of a common carotid artery of a 28 -year-old healthy volunteer was performed to evaluate the potential of the method in vivo obtaining an average SD of $4.33 \%$ over nine cardiac cycle.

The method is developed for the integration of VFI in wireless systems. The proof-of-concept feasibility of the wireless transmission and the processing in a consumer level tablet were tested, achieving a peak frame rate of 27 FPS and a data rate of $14 \mathrm{MB} / \mathrm{s}$, which is suitable for real-time flow imaging.

The limited data bandwidth required for the transmission of the first-stage LRLs makes it possible to implement a highly flexible framework, where real-time processing is firstly performed in the host system (e.g. tablet), and advanced and complex techniques can be retrospectively employed by sending the data to the cloud or to an external processing system. The velocities are simultaneously estimated everywhere 
in the VFI image, and it is possible to obtain quantitative measurements in multiple points in a multi-gated approach without any manual angle correction.

A number of limitations are given by the sequential acquisition of the flow images over $K$ emissions. First, distortions are introduced in the PSF because of the non stationarity of the medium throughout the acquisition time. Second, the long acquisition makes the method more affected by decorrelation of the scatterers. Finally, the range of detectable velocities is limited by aliasing, which depends upon the number of emissions used to create a HRI. Therefore, a tradeoff exists between the resolution of the method and the maximum detectable velocity. The velocity range can be, however, extended by using phase unwrapping methods [40].

\section{ACKNOWLEDGEMENT}

This work was supported by grant 82-2012-4 from the Danish National Advanced Technology Foundation and by BK Ultrasound.

\section{REFERENCES}

[1] C. Prinz and J. U. Voigt, "Diagnostic accuracy of a hand-held ultrasound scanner in routine patients referred for echocardiography," J. Am. Soc. Echocardiog., vol. 24, no. 2, pp. 111-116, 2011.

[2] S. Lafitte, N. Alimazighi, P. Reant, M. Dijos, A. Zaroui, A. Mignot, M. Lafitte, X. Pillois, R. Roudaut, and A. D. Maria, "Validation of the smallest pocket echoscopic device's diagnostic capabilities in the heart investigation," Ultrasound Med. Biol., vol. 37, no. 5, pp. 798-804, 2011.

[3] A. W. Kirkpatrick, M. Sirois, K. B. Laupland, D. Liu, K. Rowan, C. G. Ball, S. M. Hameed, R. Brown, R. Simons, S. A. Dulchavsky, D. R. Hamiilton, and S. Nicolaou, "Hand-held thoracic sonography for detecting post-traumatic pneumothoraces: the extended focused assessment with sonography for trauma (EFAST)," J. Trauma, vol. 57, no. 2, pp. 288-295, 2004.

[4] P. P. Sengupta, B. K. Khandheria, J. Korinek, A. Jahangir, S. Yoshifuku, I. Milosevic, and M. Belohlavek, "Left ventricular isovolumic flow sequence during sinus and paced rhythms: new insights from use of highresolution Doppler and ultrasonic digital particle imaging velocimetry," J. Am. Coll. Cardiol., vol. 49, no. 8, pp. 899-908, 2007.

[5] G.-R. Hong, G. Pedrizzetti, G. Tonti, P. Li, Z. Wei, J. K. Kim, A. Baweja, S. Liu, N. Chung, H. Houle, and et al., "Characterization and quantification of vortex flow in the human left ventricle by contrast echocardiography using vector particle image velocimetry," JACC: Cardiovascular Imaging, vol. 1, no. 6, pp. 705-717, 2008.

[6] K. L. Hansen, H. Møller-Sørensen, J. Kjaergaard, J. T. Lund, M. M. Pedersen, T. Lange, J. A. Jensen, and M. B. Nielsen, "Analysis of systolic backflow and secondary helical blood flow in the ascending aorta using vector flow imaging," Ultrasound Med. Biol., vol. 42, no. 4, pp. 899-908, 2016.

[7] M. M. Pedersen, M. J. Pihl, P. Haugaard, J. M. Hansen, K. L. Hansen, M. B. Nielsen, and J. A. Jensen, "Comparison of real-time in vivo spectral and vector velocity estimation," Ultrasound Med. Biol., vol. 38, no. 1, pp. 145-151, 2012.

[8] I. K. Ekroll, T. Dahl, H. Torp, and L. Løvstakken, "Combined vector velocity and spectral Doppler imaging for improved imaging of complex blood flow in the carotid arteries," Ultrasound Med. Biol., vol. 40, no. 7, pp. 1629-1640, 2014.

[9] S. Ricci, D. Vilkomerson, R. Matera, and P. Tortoli, "Accurate blood peak velocity estimation using spectral models and vector Doppler," IEEE Trans. Ultrason., Ferroelec., Freq. Contr., vol. 62, no. 4, pp. 686696, 2015

[10] R. W. Gill, "Measurement of blood flow by ultrasound: Accuracy and sources of error," Ultrasound Med. Biol., vol. 11, pp. 625-641, 1985.

[11] P. Peronneau, J.-P. Bournat, A. Bugnon, A. Barbet, and M. Xhaard, "Theoretical and practical aspects of pulsed doppler flowmetry real-time application to the measure of instantaneous velocity profiles in vitro and in vivo," in Cardiovascular applications of ultrasound, R. Reneman, Ed North Holland Publishing, , 1974, pp. 66-84.
[12] G. E. Trahey, J. W. Allison, and O. T. von Ramm, "Angle independent ultrasonic detection of blood flow," IEEE Trans. Biomed. Eng., vol. BME-34, no. 12, pp. 965-967, 1987.

[13] J. Bercoff, M. Tanter, L. Sandrin, S. Catheline, and M. Fink, "Ultrafast compound imaging for 2-D displacement vector measurements: application to transient elastography and color flow mapping," in Proc. IEEE Ultrason. Symp., 2001, pp. 1619-1622.

[14] B. Y. Yiu, S. S. Lai, and A. C. Yu, "Vector projectile imaging: timeresolved dynamic visualization of complex flow patterns." Ultrasound Med. Biol., vol. 40, no. 9, pp. 2295-2309, sept 2014.

[15] M. Lenge, A. Ramalli, P. Tortoli, C. Cachard, and H. Liebgott, "Planewave transverse oscillation for high-frame-rate 2-D vector flow imaging," IEEE Trans. Ultrason., Ferroelec., Freq. Contr., vol. 62, no. 12, pp. 2126-2137, December 2015.

[16] J. A. Jensen and P. Munk, "A new method for estimation of velocity vectors," IEEE Trans. Ultrason., Ferroelec., Freq. Contr., vol. 45, no. 3 , pp. 837-851, 1998.

[17] M. E. Anderson, "Multi-dimensional velocity estimation with ultrasound using spatial quadrature," IEEE Trans. Ultrason., Ferroelec., Freq. Contr., vol. 45, pp. 852-861, 1998.

[18] J. A. Jensen, "A new estimator for vector velocity estimation," IEEE Trans. Ultrason., Ferroelec., Freq. Contr., vol. 48, no. 4, pp. 886-894, 2001.

[19] H. Liebgott, A. Basarab, P. Gueth, C. Cachard, and P. Delachartre, "Lateral RF image synthesis using a synthetic aperture imaging technique." IEEE Trans. Ultrason., Ferroelec., Freq. Contr., vol. 55, no. 9, pp. $2097-$ $2103,2008$.

[20] C. Sumi, "Displacement vector measurement using instantaneous ultrasound signal phase - multidimensional autocorrelation and Doppler methods," IEEE Trans. Ultrason., Ferroelec., Freq. Contr., vol. 55, no. 1, pp. $24-43,2008$.

[21] J. A. Jensen, "Directional transverse oscillation vector flow estimation," IEEE Trans. Ultrason., Ferroelec., Freq. Contr., vol. 63, p. Submitted, 2016.

[22] S. I. Nikolov and J. A. Jensen, "In-vivo synthetic aperture flow imaging in medical ultrasound," IEEE Trans. Ultrason., Ferroelec., Freq. Contr., vol. 50, no. 7, pp. 848-856, 2003.

[23] J. Kortbek, J. A. Jensen, and K. L. Gammelmark, "Sequential beamforming for synthetic aperture imaging," Ultrasonics, vol. 53, no. 1, pp. $1-16,2013$.

[24] T. Di Ianni, M. C. Hemmsen, J. P. Bagge, H. Jensen, N. Vardi, and J. A. Jensen, "Analog gradient beamformer for a wireless ultrasound scanner," in Proc. SPIE Med. Imag., vol. 9790, 2016, pp. 979 010-1 - 8.

[25] T. Di Ianni, M. C. Hemmsen, P. L. Muntal, I. H. Jørgensen, and J. A. Jensen, "System-level design of an integrated receiver front end for a wireless ultrasound probe," IEEE Trans. Ultrason., Ferroelec., Freq. Contr., vol. 63, no. 11, pp. 1935-1946, 2016.

[26] C. Passmann and H. Ermert, "A 100-MHz ultrasound imaging system for dermatologic and ophthalmologic diagnostics," IEEE Trans. Ultrason., Ferroelec., Freq. Contr., vol. 43, pp. 545-552, 1996.

[27] C. H. Frazier and W. D. O'Brien, "Synthetic aperture techniques with a virtual source element," IEEE Trans. Ultrason., Ferroelec., Freq. Contr., vol. 45, no. 1, pp. 196-207, 1998.

[28] M. H. Bae and M. K. Jeong, "A study of synthetic-aperture imaging with virtual source elements in B-mode ultrasound imaging systems," in IEEE Trans. Ultrason., Ferroelec., Freq. Contr., vol. 47, 2000, pp. 1510-1519.

[29] Y. Li and J. A. Jensen, "Synthetic aperture flow imaging using dual stage beamforming: Simulations and experiments," J. Acoust. Soc. Am., vol. 133, no. 4, pp. 2014-2024, 2013.

[30] M. C. Hemmsen, L. Lassen, T. Kjeldsen, J. Mosegaard, and J. A. Jensen, "Implementation of real-time duplex synthetic aperture ultrasonography," in Proc. IEEE Ultrason. Symp., 2015, pp. 1-4.

[31] T. Di Ianni, M. C. Hemmsen, and J. A. Jensen, "Vector velocity estimation for portable ultrasound using directional transverse oscillation and synthetic aperture sequential beamforming," in Proc. IEEE Ultrason. Symp., 2016, pp. 1-4.

[32] J. Udesen and J. A. Jensen, "Investigation of Transverse Oscillation Method," IEEE Trans. Ultrason., Ferroelec., Freq. Contr., vol. 53, pp. 959-971, 2006

[33] J. A. Jensen, H. Holten-Lund, R. T. Nilsson, M. Hansen, U. D. Larsen, R. P. Domsten, B. G. Tomov, M. B. Stuart, S. I. Nikolov, M. J. Pihl, Y. Du, J. H. Rasmussen, and M. F. Rasmussen, "Sarus: A synthetic aperture real-time ultrasound system," IEEE Trans. Ultrason., Ferroelec., Freq. Contr., vol. 60, no. 9, pp. 1838-1852, September 2013. 
[34] J. M. Hansen, M. C. Hemmsen, and J. A. Jensen, "An object-oriented multi-threaded software beamformation toolbox," in Proc. SPIE Med. Imag., vol. 7968, March 2011, pp. 79 680Y-1-79 680Y-9.

[35] J. A. Jensen, Estimation of Blood Velocities Using Ultrasound: A Signal Processing Approach. New York: Cambridge University Press, 1996.

[36] C. A. Villagomez-Hoyos, M. B. Stuart, T. Bechsgaard, M. B. Nielsen, and J. A. Jensen, "High frame rate synthetic aperture vector flow imaging for transthoracic echocardiography," in Proc. SPIE Med. Imag., 2016.

[37] C. A. Villagomez-Hoyos, "Synthetic aperture vector flow imaging," Ph.D. dissertation, Technical University of Denmark, 2016. [Online]. Available: http://findit.dtu.dk/en/catalog/2347162876

[38] FDA, "Information for manufacturers seeking marketing clearance of diagnostic ultrasound systems and transducers," Center for Devices and Radiological Health, United States Food and Drug Administration, Tech. Rep., 2008.

[39] J. A. Jensen, M. F. Rasmussen, M. J. Pihl, S. Holbek, C. A. VillagomezHoyos, D. P. Bradway, M. B. Stuart, and B. G. Tomov, "Safety assessment of advanced imaging sequences, I: Measurements," IEEE Trans. Ultrason., Ferroelec., Freq. Contr., vol. 63, no. 1, pp. 110-119, 2016.

[40] X. Lai, H. Torp, and K. Kristofferson, "An extended autocorrelation method for estimation of blood velocity," IEEE Trans. Ultrason., Ferroelec., Freq. Contr., vol. 44, no. 6, pp. 1332-1342, 1997. 